\title{
The Effect of Unresolved Binaries on the Low-Mass IMF
}

\author{
Oleg Malkov \\ Institute of Astronomy of the Russian Acad. Sci., 48 Pyatnitskaya St., \\ Moscow 109017, Russia \\ Hans Zinnecker \\ Astrophysikalisches Institut Potsdam, An der Sternwarte 16, D-14482 \\ Potsdam, Germany
}

\begin{abstract}
We have carried out a thought experiment in which we generate a random pairing of objects drawn from a pre-assumed single star power-law IMF, which we call the fundamental IMF. We show how the mass function of primary stars, secondary stars (if we could resolve them) and the mass function of the total mass of systems differ from the underlying fundamental IMF. We also compare our results with observations and conclude that the fundamental IMF of subsolar mass stars could be steeper than currently believed. In other words: the low-mass turn-over of the observed ("apparent") IMF could be spurious, if the binary fraction of field stars is close to $100 \%$ (perhaps due to invisible companions).
\end{abstract}

\section{Monte Carlo Simulations}

The initial mass function (IMF) is normally not corrected for the effect that many if not most stars occur in (unresolved) binary systems. What is usually observed is the luminosity function or an HR-diagramme in which a point-like stellar object is taken to be a single star, although it may in fact be multiple.

In order to better understand how severe the effect of ignoring the binary nature of stars is on the true single object mass distribution, we have carried out Monte Carlo simulations in which random pairing of objects drawn from a preassumed single star power-law IMF (hereafter we call it fundamental IMF) are used for various binary fractions and slopes of the fundamental IMF. Then we ask how the mass function of primary stars and secondary stars (if we could resolve them) would differ from the fundamental IMF. Our estimates cover masses in the range from 0.01 till 10 (hereafter, all masses are given in solar units). As for fundamental IMFs we take different power-law IMFs.

Let stars be distributed with mass according to a fundamental IMF. Let us define it as $f(m)=d N / d m$. Let us then carry out a random pairing of objects drawn from a single fundamental IMF, and look at constructed pairs (binary systems). Let $f_{1}(m)=d N_{1} / d m$ be the primary mass function (primMF), $f_{2}(m)=d N_{2} / d m$ be the secondary mass function (sec-MF), and $f_{t}(m)=$ $d N_{t} / d\left(m_{1}+m_{2}\right)$ - the total mass (of system) function (tot-MF). Let us also define $f_{a}(m)=d N_{1} / d m+d N_{2} / d m$ to be the all components mass function (all-MF). 


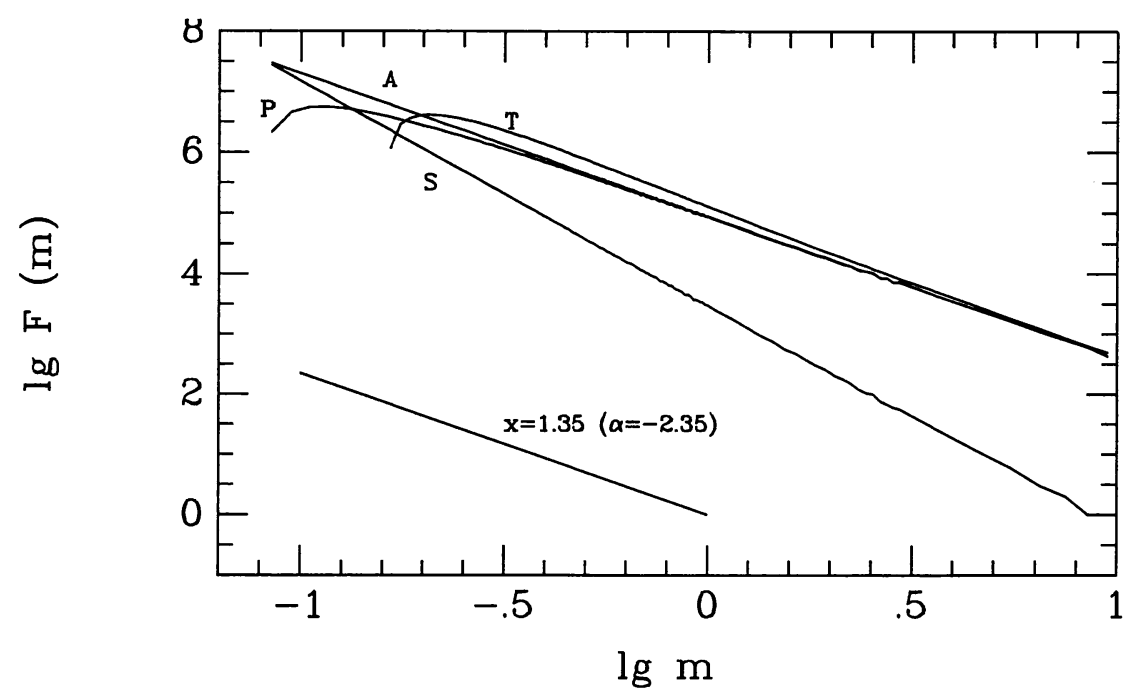

Figure 1. Random pairing of single stars from the same power-law fundamental IMF (bottom line, slope $\alpha=-2.35$, lower cut-off mass $c=0.08$ ). Initial mass functions are shown for primaries (prim-MF, $\mathrm{P}$ ), secondaries (sec-MF, S), all components together (all-MF, A) and systems (tot-MF, T, total mass of systems). Note that the all-MF reproduces the slope of the fundamental IMF.

It can be seen from Figure 1 that neither primaries, nor secondaries, nor systems mass functions are similar to the fundamental IMF. There is a natural deficiency of primaries among very low-mass stars; that is why the prim-MF is decreasing towards lower masses after some mass. Due to similar reasons (most of high-mass stars became primaries in their systems) the sec-MF is steeper than the all-MF (the all-MF is equal to the fundamental IMF in the case of random pairing).

The following conclusion can be given: there is no observational mass function (among prim-MF, sec-MF, tot-MF) that reproduces the fundamental IMF. On the contrary, the use of a power-law fundamental IMF could be an explanation for the occurrence of maxima in the observational mass function. One can recover the fundamental IMF when one observes all (both primary and secondary) components and no binaries remain unresolved $\left(f_{1}+f_{2}=2 f\right)$.

\section{Comparison with Observations}

We apply our random pairing experiments to observations and compare our results with the observational data. Let us suppose that the initial binary fraction is $100 \%$ independent of the primary mass. Assume then that pairs containig two components with masses higher the H-burning limit are all observed as binaries. Pairs with a brown dwarf (BD) secondary are observed as singles; and pairs 


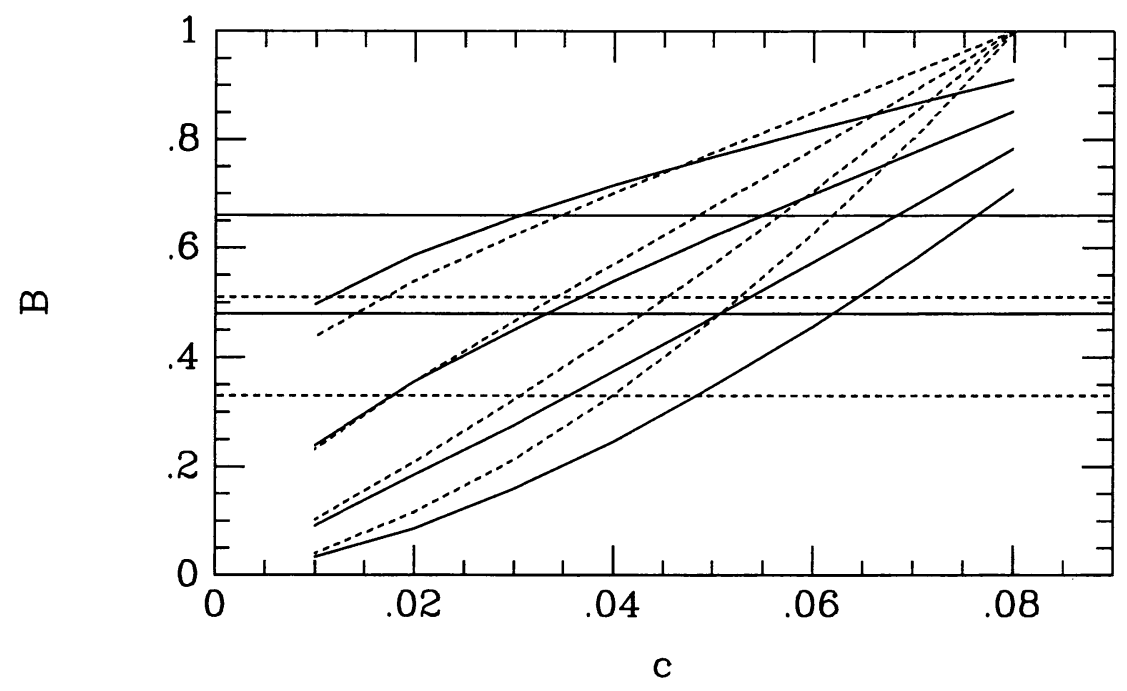

Figure 2. The observable fraction of binaries with $\mathrm{G}$ (solid lines) and $\mathrm{M}$ (dashed lines) dwarf primaries as a function of minimum mass of forming stars $c$ and of the slope of the power-law fundamental IMF (from top to bottom: $\alpha=-1.0,-1.5,-2.0,-2.5$ ). All systems are assumed to be binaries, except for pairs with a secondary mass lower than the H-burning limit, which are regarded as single stars. Horizontal lines represent observed binarity range among $\mathrm{G}$ stars found by $\mathrm{D} \& \mathrm{M}$ $(57 \% \pm 9 \%$, solid lines) and among $\mathrm{M}$ stars found by $\mathrm{F} \& \mathrm{M}(42 \% \pm$ $9 \%$, dashed lines).

with two BDs are invisible. This seems to be a good assumption for an old disk population.

The degree of observable binarity was calculated for $G$ and $M$ primaries. These results were compared with observational data for G (Duquennoy \& Mayor 1991, hereafter D\&M) and M (Fischer \& Marcy 1992, hereafter F\&M) primaries. Figure 2 illustrates the fact that the calculated binarity of $G$ stars exceeds the corresponding one of $\mathrm{M}$ stars (as observations suggest) for the flatter fundamental IMFs, with slopes $\alpha \geq-1.5$, and for low enough cut-off mass $c$.

We also calculated mass ratio distributions for different slopes of the powerlaw fundamental IMF, varying the lower limit of forming stars $c$ from 0.01 to 0.08 and compared the results with the observed mass ratio distributions for $\mathrm{G}$ stars (the D\&M set). The results of $\chi^{2}$ tests do not depend on $c$ but strongly depend on $\alpha$ : the flatter the fundamental IMF the smaller the value of $\chi^{2}$. For $\alpha=-1.0$ and $c=0.08$, Figure 3 represents the comparison for $\mathrm{G}$ primaries.

\section{Conclusions}

It is crucial to distinguish the observational mass functions of components and of the total mass of systems. The fundamental IMF can be drastically differ- 


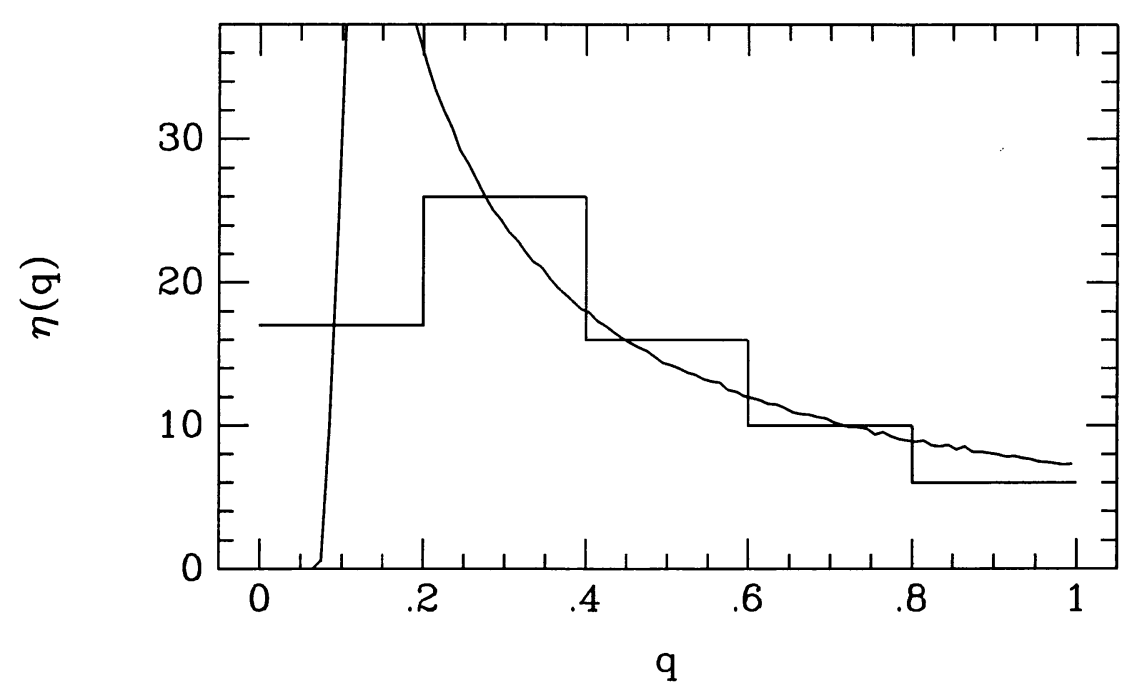

Figure 3. Binary mass ratio distribution $\eta(q)$ for random pairing of the component stars. Comparison of the calculated distribution for G dwarf primaries (curve) with observational data from D\&M (histogram). Only wide binaries $\left(\log P^{d}>4\right)$ are taken from the D\&M sample. For the best fit, the slope of the fundamental IMF is $\alpha=-1.0$, the lower mass limit is $c=0.08$. Distributions are normalized to the number of systems with $0.2<q<1$. According to D\&M, the observational bin $q=0-0.2$ is very uncertain.

ent from them: even power-law fundamental IMFs can lead to quasi-lognormal turnovers for observed mass functions uncorrected for the effect of binaries. Binary fractions for MS stars may not be very different from 100\%, judging from observations of pre-MS stars (cf. Mathieu 1994). Under this assumption and if random pairing is assumed to be correct, our predictions of observational binarity are close to published estimations. If so, the lower limit of substellar masses must be below 0.05 solar masses and depends on the slope of the fundamental IMF.

Acknowledgements OM thanks the Deutsche Forschungsgemeinschaft and the SOC of IAU Symp 200 for financial support.

\section{References}

Duquennoy, A., \& Mayor, M. 1991, A\&A, 248, 485

Fischer, D. A., \& Marcy, G. W. 1992, ApJ, 396, 178

Malkov, O., \& Zinnecker, H. 2001, MNRAS, 321, 149

Mathieu, R. 1994, ARA\&A, 32, 465 